Kansas State University Libraries

New Prairie Press

\title{
ESTIMATION OF KINETIC PARAMETERS ASSOCIATED WITH NUTRIENT UPTAKE BY AN INTACT PLANT ROOT SYSTEM
}

\author{
Edward Gbur \\ Craig Beyrouty
}

Follow this and additional works at: https://newprairiepress.org/agstatconference

Part of the Agriculture Commons, and the Applied Statistics Commons

\section{(c) (1) $\Theta$}

This work is licensed under a Creative Commons Attribution-Noncommercial-No Derivative Works 4.0 License.

\section{Recommended Citation}

Gbur, Edward and Beyrouty, Craig (1996). "ESTIMATION OF KINETIC PARAMETERS ASSOCIATED WITH NUTRIENT UPTAKE BY AN INTACT PLANT ROOT SYSTEM," Conference on Applied Statistics in Agriculture. https://doi.org/10.4148/2475-7772.1319

This is brought to you for free and open access by the Conferences at New Prairie Press. It has been accepted for inclusion in Conference on Applied Statistics in Agriculture by an authorized administrator of New Prairie Press. For more information, please contact cads@k-state.edu. 


\title{
ESTIMATION OF KINETIC PARAMETERS ASSOCIATED WITH NUTRIENT UPTAKE BY AN INTACT PLANT ROOT SYSTEM
}

\author{
Edward Gbur and Craig Beyrouty \\ University of Arkansas
}

\begin{abstract}
Several mechanistic models have been developed for the prediction of nutrient uptake at low concentrations from the soil by a plant root system. Claassen and Barber (1974 Plant Physiology 54, 564-568; 1976 Agrcriomy Journal 68, 961-964) presented an experimental procedure to obtain data from intact plants to fit an ion depletion curve and used the data in a model which they developed to predict nutrient uptake. Their model assumed that nutrient absorption from the soil solution followed Michaelis-Menten kinetics. In this paper, we develop a stochastic version of the Claassen-Barber model and illustrate its application to the estimation of the kinetic parameters associated with the uptake of potassium by corn plants. The analysis requires the fitting of a nonlinear regression equation which cannot be explicitly expressed in terms of the response variable. The analysis is potentially further complicated by heterogeneity of variance and an autocorrelated error structure.

Keywords: Nonlinear regression, Implicitly defined function, Michaelis-Menten kinetics.
\end{abstract}

\section{Introduction}

Plant scientists have studied the relationship between nutrient uptake by plants and the root environment. They have developed a number of mechanistic root-soil models to predict nutrient uptake which incorporate physical and chemical soil characteristics that influence nutrient movement in soil and uptake by roots. The models have three main components: (i) soil characteristics which affect nutrient movement, (ii) absorption characteristics of the root system, and (iii) morphology and growth characteristics of the root system.

Building on the work of Nye and Marriot (1969), Claassen and Barber (1974, 1976) developed a mechanistic model to predict nutrient uptake based on Michaelis-Menten type kinetics. Their model was later refined by Cushman (1979) to account for root competition. Similar models have been proposed by other researchers (cf., Rendig and Taylor, 1989, Chapter 5). These models have found widespread use in agronomic research despite the lack of statistical inference procedures, including 
calculation of standard errors for the kinetic parameter estimates.

Among the assumptions of the Claassen and Barber model is that absorption of nutrients by the roots is from the soil solution. This assumption allows for the estimation of absorption characteristics of the roots from plants growing in nutrient solution rather than soil. This simplifies experimental procedures since the kinetic parameters in the model can be estimated indirectly by measuring the rate of depletion of the nutrient solution over time.

In this paper we investigate a stochastic version of the influx portion of the Claassen-Barber model. The model is developed in section 2 and model fitting is discussed in section 3. Data on the uptake of potassium by corn plants are analyzed in section 4 .

\section{Derivation of the kinetic model}

Influx (uptake rate) is the amount of an ion which moves from an external nutrient solution into the roots through one unit of root surface area per unit time. Net influx (influx minus efflux) can be measured indirectly by measuring ion depletion from a nutrient solution over time.

Let $y=$ ion concentration in solution and $x=t i m e$. In the kinetic model, $y$ will be expressed as a function of $x$. Assuming that uptake is governed by Michaelis-Menten kinetics, net influx is given by

$$
I_{N}=\frac{I_{\max }\left(y-C_{\min }\right)}{K_{m}+Y-C_{\min }},
$$

where $I_{\max }=$ maximum influx,

$\mathrm{C}_{\min }=$ minimum concentration at which influx can occur,

$\mathrm{K}_{\mathrm{m}}=$ Michaelis constant.

Since $I_{N}$ is a time rate of change, it can be expressed as

$$
I_{N}=-\frac{V}{A} \frac{d y}{d x},
$$

where $\mathrm{V}=$ solution volume,

$A=$ root surface area.

Equating the expressions for $I_{N}$ from (1) and (2) leads to the differential equation

$$
\left(1+\frac{K_{n}}{Y-C_{\min }}\right) d y-\left(\frac{A I_{\max }}{V}\right) d x=0 .
$$


Since the equation is separable, term by term integration yields the deterministic kinetic model

$$
y+K_{m} \ln \left(y-C_{\min }\right)=y_{0}+K_{m} \ln \left(y_{0}-C_{\min }\right)-\left(\frac{A I_{\max }}{V}\right) x,
$$

where $y_{0}=$ concentration at time 0 . The kinetic parameters $I_{\max }$, $C_{\min }$, and $\mathrm{K}_{\mathrm{m}}$ in (3) describe the absorption characteristics of the plant's root system. Note that (3) can not be explicitly written in the form $y$ equals some function of $x$.

A graph of solution concentration as a function of time is commonly referred to as a depletion curve and is visually similar to an exponential decay having a horizontal asymptote at $\mathrm{y}=\mathrm{C}_{\min }$. The value of $C_{\min }$ in (3) is influenced primarily by time points at the end of the depletion. $I_{\max }$ is the maximum of the slopes of the tangent lines to the function and its value is influenced primarily by time points at the beginning of the depletion. $\mathrm{K}_{\mathrm{m}}$ is the concentration at which the slope is one-half of its maximum value and is influenced primarily by time points in the "middle" of the depletion.

The kinetic model (3) can be rewritten in more familiar regression type notation as

$$
y+\beta_{1} \ln \left(y-\beta_{2}\right)=\beta_{0}-\beta_{3} x,
$$

where $\beta_{0}=\mathrm{y}_{0}+\mathrm{K}_{\mathrm{m}} \ln \left(\mathrm{y}_{0}-\mathrm{C}_{\min }\right), \quad \beta_{1}=\mathrm{K}_{\mathrm{m}}, \quad \beta_{2}=\mathrm{C}_{\min }$, and $\beta_{3}=A I_{\max } / \mathrm{V}$.

A stochastic version of (4) can be written as

$$
y=g(x ; \beta)+\epsilon,
$$

where $\epsilon$ is random error and the nonlinear regression function $g(x, \beta)$ can be written implicitly but not explicitly from (4). The $\epsilon$ are assumed to be independent $N\left(0, \sigma^{2}\right)$. By definition, the coefficients are subject to the constraints

$$
\beta_{1}>0, \beta_{2}>0, \beta_{3}>0 \text {. }
$$

In addition, from the definition of $C_{\min }, y>\beta_{2}$. Note that the ion concentration, $y$, is stochastic and the time, $x$, is nonstochastic.

\section{Model fitting}

Given data from a depletion curve, estimation of $\beta$ in (5) can be accomplished by nonlinear least squares, which requires the minimization of 


$$
\mathrm{S}(\beta)=\sum_{\mathrm{i}}\left(y_{\mathrm{i}}-\mathrm{g}\left(\mathrm{x}_{\mathrm{i}} ; \beta\right)\right)^{2}
$$

with respect to $\beta$. In addition to the usual nonlinear least squares issues of starting values for $\beta$ and partial derivatives of $g(x ; \beta)$, we need to consider how to compute values of $g(x ; \beta)$ for given $x$ and $\beta$. Each of these issues will be discussed in turn.

\section{(i) Computing $g(x ; \beta)$ :}

Based on (4), define the function

$$
G(y ; x, \beta)=y+\beta_{1} \ln \left(y-\beta_{2}\right)-\beta_{0}+\beta_{3} x \text {, for } y>\beta_{2} \text {. }
$$

Given $\mathrm{x}$ and $\beta$, solving the equation $\mathrm{G}(\mathrm{y})=0$ for $\mathrm{y}$ is the same as solving (4) for $y$, which is the same as finding the value of $g(x ; \beta)$ in $(6)$.

For $\beta_{\mathrm{i}}>0, i=1,2,3$, the following properties of $G(y ; x, \beta)$ can be derived using straightforward calculus techniques.

(a) For fixed $x$ and $\beta, G(y ; x, \beta)$ is a strictly increasing and concave function of $y$, for $y>\beta_{2}$.

(b) For fixed $y$ and $\beta, G(y ; x, \beta)$ is an increasing function of $x$.

(c) For sufficiently large initial concentrations $y_{0}$, $\mathrm{G}\left(\mathrm{Y}_{0} ; 0, \beta\right)>0$. An example of a sufficient condition is $y_{0}>\max \left\{\beta_{0}, \beta_{2}+1\right\}$.

(d) $\lim _{y \rightarrow \beta_{2}^{*}} G(y ; x, \beta)=-\infty$, $\lim _{y \rightarrow+\infty} G(Y ; x, \beta)=+\infty$.

Using the above properties, the equation $G(y)=0$ has a unique root which is a decreasing function of $x$ with limit $\beta_{2}$. To find the root, we can use Newton's method and shorten the search interval for each $x$ within each iteration of the nonlinear least squares routine.

The importance of the above properties of $G(y ; x, \beta)$ should not be underestimated. The equation $G(Y)=0$ must be solved for each $\mathrm{x}$ at each iteration of the nonlinear least squares routine. For example, if there were 50 time points in the depletion and there were an average of 10 iterations in the minimization, then $G(y)=0$ would need to be solved 500 times for each set of starting values for $\beta$. If 4 sets of starting values were used and there were 10 experimental units (and hence, depletion curves) in the experiment, then $G(y)=0$ would have been solved 20,000 times. 
(ii) Derivatives of $g(x ; \beta)$ :

Since $g(x ; \beta)$ is implicitly defined, the partial derivatives of $g(x ; \beta)$ with respect to each $\beta_{i}$ can be obtained using implicit differentation techniques. The derivatives are as follows:

$$
\begin{aligned}
& \frac{\partial g(x ; \beta)}{\partial \beta_{0}}=\frac{g(x ; \beta)-\beta_{2}}{\beta_{1}+g(x ; \beta)-\beta_{2}}, \\
& \frac{\partial g(x ; \beta)}{\partial \beta_{1}}=-\frac{\partial g(x ; \beta)}{\partial \beta_{0}}\left[\ln \left(g(x ; \beta)-\beta_{2}\right)\right], \\
& \frac{\partial g(x ; \beta)}{\partial \beta_{2}}=\frac{\beta_{1}}{\beta_{1}+g(x ; \beta)-\beta_{2}}, \\
& \frac{\partial g(x ; \beta)}{\partial \beta_{3}}=-\frac{\partial g(x ; \beta)}{\partial \beta_{0}} x .
\end{aligned}
$$

(iii) starting values for $\beta$ :

one method of obtaining starting values for $\beta$ is to solve

(4) for $x$ to get

$$
x=\alpha_{0}+\alpha_{1} y+\alpha_{2} \ln \left(y-\beta_{2}\right),
$$

where

$$
\alpha_{0}=\frac{\beta_{0}}{\beta_{3}}, \quad \alpha_{1}=-\frac{1}{\beta_{3}}, \quad \alpha_{2}=-\frac{\beta_{1}}{\beta_{3}} .
$$

An inital value for $\beta_{2}$ can be obtained from a data plot by graphically estimating the horizontal asymptote. Using this initial value for $\beta_{2}$, multiple linear regression can be used to estimate the $\alpha^{\prime} s$ and in turn, the remaining $\beta_{i}^{\prime} s$. Recall that $x$ (time) is not stochastic so that the estimated $\beta_{i}{ }^{\prime}$ s do not have their usual statistical properties.

\section{Numerical example}

Unpublished data (courtesy of Dr. Moshe Silberbush) on potassium uptake by corn plants will be used to illustrate the estimation of the kinetic parameters in the model. Details on experimental methods for this type of experiment can be found in Claassen and Barber (1974).

Corn plants were grown in 4 liter pots, 4 plants per pot, with continuously aerated nutrient solution. After 19 days, the plants were starved of potassium for 24 hours prior to data collection. A time 0 , the plants were tranferred to a nutrient solution to which $100 \mu \mathrm{M} / \mathrm{L}$ of potassium had been added. The 
solution was sampled continuously but bulked into $3 \mathrm{ml}$ samples based on 5 minute time intervals. The samples were analyzed for potassium concentration by atomic absorption spectrophotometry. Immediately following the completion of sampling, root length and mean root radius were measured and root surface area was calculated under the assumption that the roots were smooth cylinders. The root surface area and the initial solution volume will be assumed to be known constants.

The data are listed in Table 1 and plotted in Figure 1. The first two observations were discarded for reasons related to the startup of the depletion. From the figure the concentration at which the horizontal asymptote occurs is not obvious. A grid of values for $\beta_{2}\left(C_{\min }\right)$ was used in (7) to obtain sets of starting values for the nonlinear least squares procedure. These sets of starting values are listed in Table 2. Some agronomists have used (7) with visual estimates of $\beta_{2}$ from the data plot to obtain kinetic parameter estimates. Table 2 indicates that the estimates of $\beta_{1}$ and $\beta_{3}$ (and hence, $K_{m}$ and $I_{\max }$ ) vary considerably depending on the "eyeballed" estimate for $\beta_{2}$.

SAS's NLIN procedure was used to carry out the nonlinear least squares estimation. The NLIN statements are given in the Appendix. The nonlinear least squares procedure converged to the same minimum for all sets of starting values in Table 2 except for $\beta_{2}=0.5$, which failed to converge after 50 iterations. The fitted model is given by

$$
\hat{y}+38.001 \ln (\hat{y}-1.995)=271.183-0.994 x,
$$

with SSE $=111.636$.

A plot of the studentized residuals versus time is shown in Figure 2. Since it was anticipated that the data might be autocorrelated, the $i^{\text {th }}$ residual was plotted against the $(i-1)^{\text {st }}$ residual (Figure 3 ). The estimated autocorrelation coefficient was $\hat{\rho}=0.443$ with a p-value of 0.0033 , indicating possible autocorrelation.

Upon investigating the three consecutive large negative residuals beginning at $x=110$, it was determined that the spectrophotometer had been recalibrated for samples collected after time $\mathrm{x}=130$ to compensate for the lower potassium solution concentrations. The concentrations for observations immediately preceeding recalibration were near the detection limit of the original calibration and it was thought that they might be unreliable. The observations at $x=110,115$, and 120 were deleted and the model was refit. The observations at $\mathrm{x}=125$ and 130 were judged to be outliers in the refitted model. The new outliers were removed and the model was refit again for sets of 
starting values with $\beta_{2}$ ranging from 1.0 to 2.5 . In each case, the nonlinear least squares procedure converged to the same minimum. The fitted model without outliers is given by

$$
\hat{Y}+37.234 \ln (\hat{y}-0.881)=266.405-0.939 x ，
$$

with SSE $=40.363$.

A plot of the studentized residuals versus time is shown in Figure 4. It appears that the variance is smaller after recalibration of the spectrophotometer than before. Variances were estimated for each time period separately, yielding estimates of 1.746 before and 0.460 after recalibration. Comparing their ratio 3.79 to an F-distribution with 18 and 19 df gives a p-value of 0.0059. Although the p-value is not exact since the estimated variances are not independent, it is reasonable to conclude that the variances are not equal.

The plot of the $i^{\text {th }}$ versus $(i-1)^{\text {st }}$ residual did not indicate autocorrelation. The estimated autocorrelation for the entire depletion was $\hat{\rho}=0.099$. The estimated autocorrelation before recalibration was $\hat{\rho}=-0.208$ and after recalibration was $\hat{\rho}=$ 0.006. Thus, with the outliers removed, there appears to be no autocorrelation in the residuals. The data points which were removed as outliers in the refitted model are the extreme points in the third quadrant in Figure 3 so that the lack of significant autocorrelation is not suprising.

A possible physical explanation for the lack of significant autocorrelation is that the relatively large time interval used when bulking the solution samples eliminates any time dependency. This explanation is consistent with preliminary data from another study in which samples from small time intervals were combined into larger intervals. Indications of strong autocorrelation disappeared as the length of the time interval increased.

In the final model fit, we shall assume that there is no autocorrelation. A final weighted nonlinear least squares fit with empirical weights in the ratio of 3.8 to 1 produced the estimated coefficients and their estimated asymptotic standard errors and correlations given in Table 3. Note the strong correlations among the estimated parameters. Based on the estimates in Table 3, the estimated kinetic parameters are

$$
\begin{aligned}
& \hat{\mathrm{C}}_{\min }\left(=\beta_{2}\right)=1.07 \mu \mathrm{M} / \mathrm{L}, \\
& \hat{\mathrm{K}}_{\mathrm{m}}\left(=\beta_{1}\right)=34.11 \mu \mathrm{M} / \mathrm{L}, \\
& \hat{\mathrm{I}}_{\max }\left(=\mathrm{V} \beta_{3} / \mathrm{A}\right)=0.001017 \mu \mathrm{M} / \mathrm{cm}^{2} \mathrm{~min}=0.17 \mu \mathrm{M} / \mathrm{m}^{2} \mathrm{sec} .
\end{aligned}
$$


The estimated asymptotic standard error of $\hat{I}_{\max }$ is $0.01 \mu \mathrm{M} / \mathrm{m}^{2} \mathrm{sec}$. Residual plots from this fit did not indicate any problems with unequal variance or autocorrelation.

The kinetic parameter estimates are in general agreement with values reported previously in the literature (e.g., Barber, 1984, Chapter 10). The large estimated standard error for $\mathrm{C}_{\min }$ may simply be a reflection of the information content of the data. From the graphical interpretation of the kinetic parameters given in section 2 , the information on $C_{\min }$ is concentrated in the "final" portion of the depletion. Since the horizontal asymptote is not clearly defined in Figure 1, the data contain little information from which $C_{\min }$ can be estimated accurately.

\section{Conclusion}

The stochastic model developed in section 2 from the mechanistic model of Claassen and Barber provides a sound basis from which estimates and approximate standard errors of kinetic parameters can be obtained by common statistical methods. Using this stochastic model, the effects of various factors on the kinetic parameters can be compared and the ability of the claassen-Baraber model to predict nutrient uptake can be evaluated statistically under specified conditions. This has not been possible previously.

The numerical results presented in section 4, while limited in scope, point out that the methods used previously in the subject matter literature have serious shortcomings. Our results demonstrate that the choice of an "eyeball" estimate of $\mathrm{C}_{\min }$ from a depletion curve graph can have a dramatic effect on the estimates of $K_{m}$ and $I_{\max }$. While this is not suprising given the strong correlations among the estimates, it has not been clearly recognized in the literature. The large standard errors for some parameter estimates have implications for the design of depletion studies. Experimenters generally design depletions to be terminated when it is thought a priori that uptake will cease. In our results, the final estimate of $C_{\min }$ was approximately onefourth of the smallest (and last) observation in the depletion. Thus, our numerical results indicate a need for research into the design of depletion studies of this type.

\section{References}

Barber, S. A. (1984). Soil Nutrient Bioavailability. A Mechanistic Approach. New York: John Wiley \& Sons.

Claassen, N. and Barber, S. A. (1974). A method for characterizing the relation between nutrient concentration 
and flux into roots of intact plants. Plant Physiology 54, 564-568.

Claassen, N. and Barber, S. A. (1976). Simulation model for nutrient uptake from soil by a growing plant root system. Agronomy Journal 68, 961-964.

Cushman, J. H. (1979). An analytical solution to solute transport near root surface for low initial concentration. I. Equation development. Soil Science Society of America Journal 43, 1087-1092.

Nye, P. H. and Marriott, F. H. C. (1969). A theoretical study of the distribution of substances around roots resulting from simultaneous diffusion and mass flow. Plant and Soil 30 , 459-472.

Rendig, V. V. and Taylor, H. M. (1989). Principles of Soil-Plant Interrelationships. New York: McGraw-Hill.

Table 1. Data from an experiment on potassium uptake by corn.

\begin{tabular}{cccccc} 
Time* & $\begin{array}{c}\text { Potassium } \\
\text { concentration }\end{array}$ & Time & $\begin{array}{c}\text { Potassium } \\
\text { concentration }\end{array}$ & $\begin{array}{c}\text { Potassium } \\
\text { concentration }\end{array}$ \\
\hline 15 & 87.1 & 95 & 38.6 & 170 & 13.4 \\
20 & 83.0 & 100 & 38.6 & 175 & 11.5 \\
25 & 79.0 & 105 & 34.5 & 180 & 10.8 \\
30 & 75.0 & 110 & 28.5 & 185 & 10.8 \\
35 & 75.0 & 115 & 26.4 & 190 & 7.85 \\
40 & 72.9 & 120 & 24.4 & 195 & 7.59 \\
45 & 66.9 & 125 & 24.4 & 200 & 7.34 \\
50 & 66.9 & -- Recalibration-- & 205 &. \\
55 & 62.8 & 130 & 21.2 & 210 &. \\
60 & 56.8 & 135 & 23.1 & 215 & 6.30 \\
65 & 56.8 & 140 & 23.1 & 220 & 5.01 \\
70 & 52.7 & 145 & 20.5 & 225 & 4.50 \\
75 & 50.7 & 150 & 19.9 & 230 & 4.24 \\
80 & 46.7 & 155 & 16.6 & 235 & 4.24 \\
85 & 46.7 & 160 & 15.9 & 240 & 4.11 \\
90 & 42.6 & 165 & 15.2 & & \\
\hline$*$ & The table entries are potassium solution concentration \\
( $\mu$ M/L) and time (min). The initial solution volume was 4 L and \\
the estimated root surface area was 3540 cm ${ }^{2}$. Recalibration of \\
the spectrophotometer used to measure potassium concentration \\
occurred prior to analysis of samples taken after 130 min.
\end{tabular}




\section{Table 2. Sets of starting values for the initial nonlinear least squares fit for selected values of $\beta_{2}$.}

\begin{tabular}{cccc}
$\beta_{2}$ & $\underline{\beta}_{0}$ & $\beta_{1}$ & $\beta_{3}$ \\
\hline 0.5 & 344.321 & 53.442 & 1.164 \\
1.0 & 308.888 & 46.119 & 1.081 \\
1.1 & 302.144 & 44.728 & 1.065 \\
1.2 & 295.595 & 43.376 & 1.049 \\
1.3 & 289.231 & 42.056 & 1.034 \\
1.4 & 283.043 & 40.771 & 1.019 \\
1.5 & 276.746 & 39.476 & 1.004 \\
2.0 & 247.115 & 33.368 & 0.931 \\
2.5 & 219.344 & 27.662 & 0.861 \\
3.0 & 192.597 & 22.190 & 0.790 \\
\hline
\end{tabular}

Table 3. Parameter estimates and estimated asymptotic standard errors and correlations from the weighted nonlinear least squares fit to the model.

\begin{tabular}{cccccc} 
& & \multicolumn{2}{c}{ Estimated } & \multicolumn{2}{c}{ Estimated correlation } \\
Parameter & Estimate & standard error & $\beta_{0}$ & $\beta_{1}$ & $\beta_{2}$ \\
\hline$\beta_{0}$ & 251.46 & 34.09 & -- & & \\
$\beta_{1}$ & 34.11 & 7.19 & 0.999 & -- & \\
$\beta_{2}$ & 1.07 & 0.97 & -0.884 & -0.888 & -- \\
$\beta_{3}$ & 0.90 & 0.08 & 0.991 & 0.989 & -0.824 \\
\hline
\end{tabular}




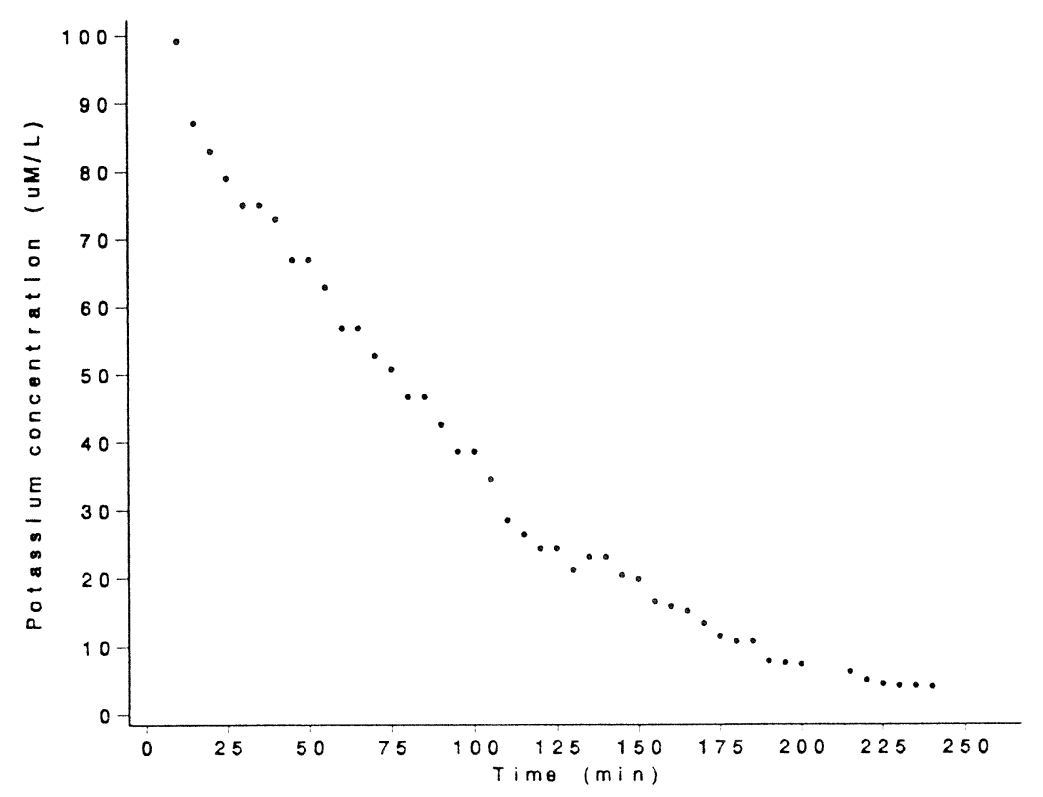

Figure 1. Potassium concentration in the nutrient solution as a function of time.

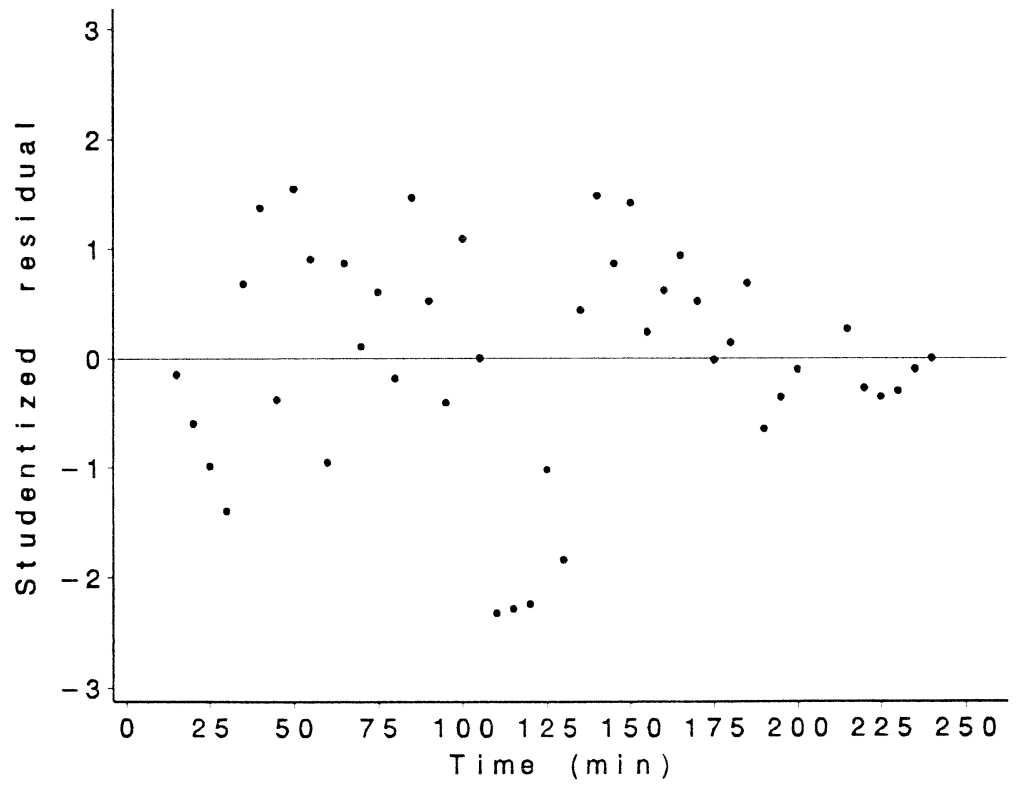

Figure 2. Studentized residuals from the initial fit of the stochastic model (5). 


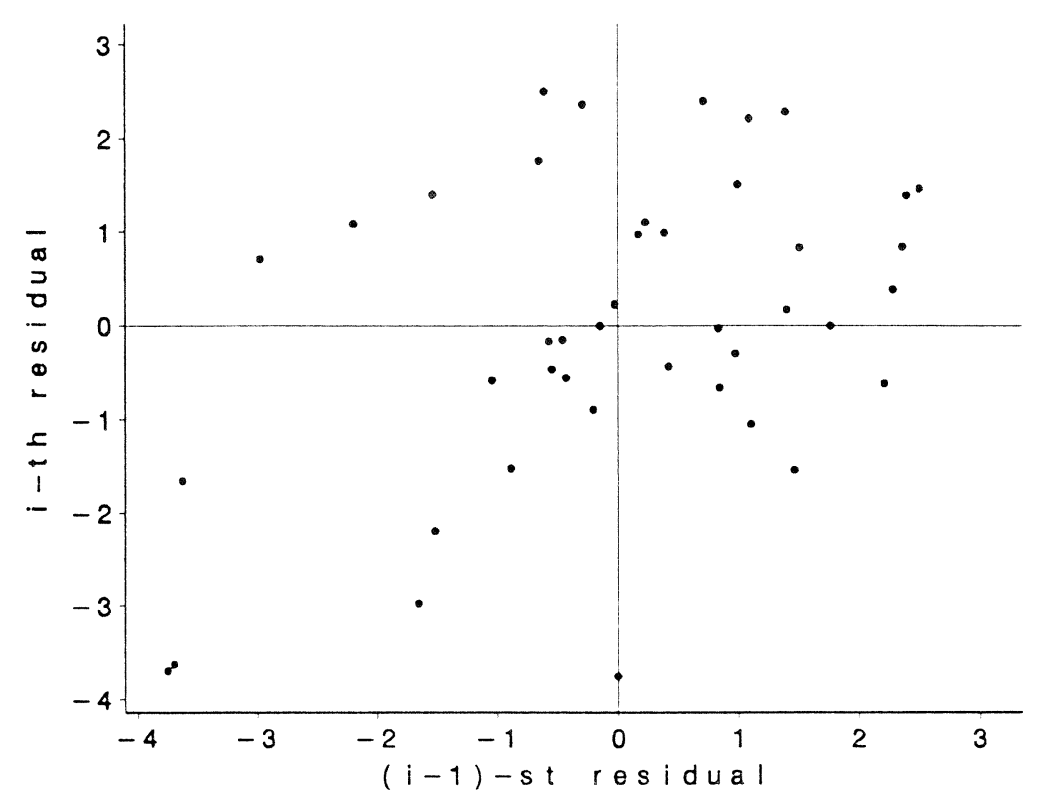

Figure 3. Residuals versus lagged residuals from the initial fit of the stochastic model (5).

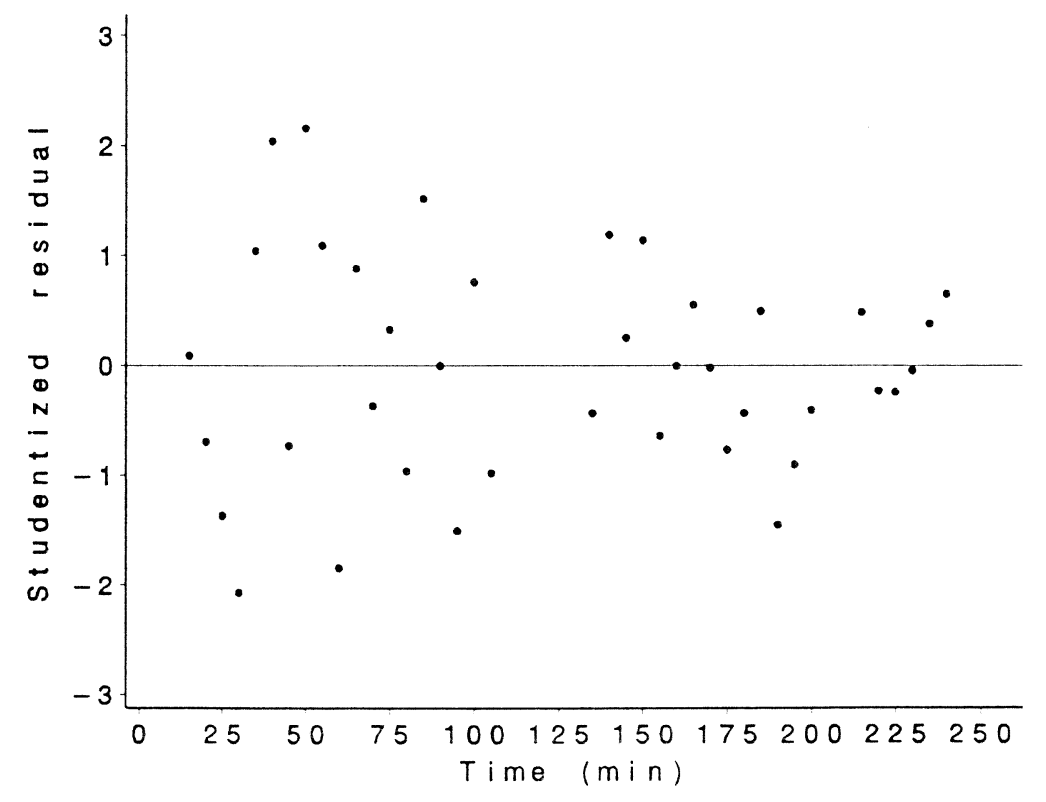

Figure 4. Studentized residuals from the fit of the stochastic model (5) with all outliers from the initial fit removed. 


\section{Appendix}

The following SAS PROC NLIN code was used to fit the model (5).

proc nlin data $=$ corn method=Marquardt $g 4$ best $=5$;

bounds $\mathrm{b} 1>0, \mathrm{~b} 2>0, \mathrm{~b} 3>0$;

parms bo $=295.0$ to 296.0 by .5

$\mathrm{b} 1=43.0$ to 43.5 by 0.25
$\mathrm{~b} 2=1.2$
$\mathrm{~b} 3=1.02$ to 1.06 by $.02 ;$

* Values used for */
$/$ * illustration

/* Newton's method for solving $G(Y)=0$ */

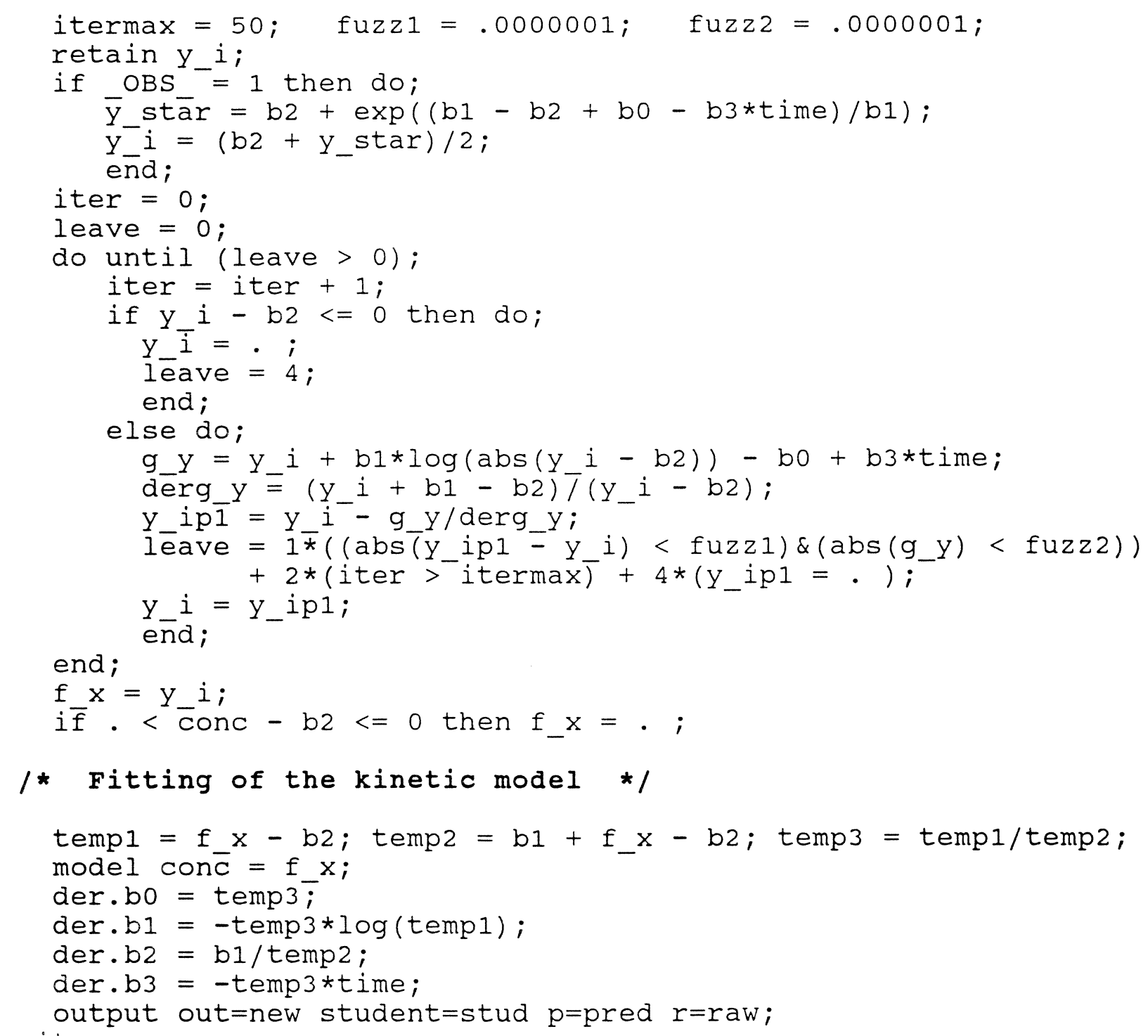

